\title{
REVIEWS
}

The Acoustic Bubble. By T. G. Leighton. Academic Press, 1994. 613 pp. £95. ISBN 0-12-441920-8.

The dynamics of bubbles in liquids are relevant in a wide variety of fields. Collapsing cavitation bubbles erode marine propellers and hydraulic machinery. Gas-liquid twophase flows are important in many process, power and extractive industrial contexts. The acoustic properties of liquids are dramatically affected by the presence of bubbles: the velocity of sound can drop to less than $5 \%$ of its bubble-free value. Similarly, acoustic fields can also dramatically affect bubbles. These and many other effects and applications appear, at least briefly, in this book.

At first sight The Acoustic Bubble appears to be a substantial research monograph; however, the reader soon discovers the author has much wider aims. The book has many of the properties of a textbook (both introductory and advanced), a general introduction, and an encyclopaedic reference work (over 1200 references) as well as those of a research monograph. This can be irritating if readers do not take account of the author's introductory warning that 'individual readers will encounter sections that are too rudimentary or too involved, depending on their background'. However, the author's main intention is to give "the reader a 'physical feel' for the acoustic interactions of acoustic fields with bubbles". There are plenty of diagrams and photographs with many, appropriate, references to the author's own work.

The book is divided into five substantial chapters; only the first two are less than 100 pages long. The first chapter, on 'The sound field', has a large introductory component but passes on to aspects of underwater ultrasound including nonlinear effects, acoustic streaming, and parametric and stimulated scattering effects.

Chapter 2 on 'Cavitation inception and fluid dynamics' has a good discussion of the first topic and the collapsing spherical cavity. The introduction to fluid mechanics is soon followed by discussion of the moving bubble with a rather brief survey of moving and deformed bubbles.

The major topic of the book is approached through chapter 3 on 'The freelyoscillating bubble'. This contains much more than the linear modes of oscillation of an isolated bubble. Acoustic radiation from a bubble including the case of a nearby boundary is presented, and, as for most topics, details of some practical cases are included. The contributions of radiation, thermal diffusion and viscosity to damping are discussed. Bubble growth and separation on injection from a nozzle is discussed in depth. Natural bubble entrainment gets a large section including the babbling brook, and many aspects of the noise of rain on water, where bubble entrainment is significant for sound production. Wind effects, through wave-breaking, get a much briefer treatment which is offset by the subsequent large section on bubbles in the ocean.

Sound pressure fields and bubbles are brought together in chapter 4, 'The forced bubble'. Although linear aspects, resonance, velocity of sound, etc. are discussed, most attention is given to nonlinear motions and strong forcing fields, mostly with regard to ultrasonic frequencies. These fields can generate strongly negative pressures so that there is much discussion of cavitation, which can be stable or transient. Stable cavitation describes bubbles that after initial transients settle to large-amplitude periodic oscillations whereas the bubbles of transient cavitation evolve, perhaps with oscillations, until they collapse catastrophically. There is not a clear-cut distinction 
since many longer-term or larger-scale features can affect bubble evolution, but it serves to guide the exposition and many details are given. A major topic is the transfer of gas between bubble and liquid. Ordinarily bubbles eventually dissolve, but in the presence of a sound field 'rectified diffusion' can occur for large enough bubbles. There are therefore a number of possible evolutionary developments for bubbles generated by or pre-existing in a sound field and these are discussed in detail.

The final chapter 'Effects and mechanisms' has three main topics. Bubble detection and cavitation and its effects are natural developments of the foregoing chapters, but include much discussion of the various practical aspects of applications, for example the effects of ultrasound on biological tissues, where cavitation may be important. This is a topic that is also mentioned elsewhere, giving the impression that the author hopes that he has made the book accessible to many clinical users of ultrasound.

The other main topic of the final chapter may come as a surprise to many readers of the book. 'Sonoluminescence' is the emission of light by bubbles oscillating in a sound field. It is stronger for more rapid contraction of bubbles and appears to be related to the high temperatures and pressures that may occur, e.g. temperatures such as $5000 \mathrm{~K}$. There has been, and is, controversy and uncertainty over the details of the light emission. High temperature, free radicals, electric charges, and imploding shock waves all figure in one scenario or another. Further questions are raised by perfectly periodic light emission by a single bubble in experiments which show a light pulse so short that, to date, only ever-lower upper bounds on its duration have been found. (See 1993 IUTAM Symposium on 'Bubbles and interface phenomena'.)

For a book of this size commendably few misprints and errors were noticed. It would have helped if the printers had not chosen a font in which italic 'vee' and Greek 'nu' are almost indistinguishable. I am not convinced that aiming at a wide audience with one comprehensive book is better than three more carefully targeted books, but it does have some advantages of completeness.

In conclusion, I congratulate the author on producing such as comprehensive book, and suggest that a number of readers of this journal will find it to be of sufficient value to justify its purchase. In addition, it is a work of reference that many libraries should stock.

D. H. Peregrine

\section{Wave Propagation in Gas-Liquid Media. By V. E. Nakoryakov, B. G. Pokusaev and I. R. SHreIBER. CRC Press, 1993. 222 pp. ISBN 0-8493-9906-8.}

This book summarizes work done in the period 1970-1984 on waves in gas/liquid media. Emphasis is put on the experimental and theoretical work of the authors and their colleagues in the Institute of Thermophysics of the Siberian branch of the Academy of Science, at Novosibirsk, under the directorship of S. S. Kutateladze.

A first edition, in Russian, appeared in 1983 followed by a second one in 1990. The English edition is a translation of the first Russian one, and references only go up to about 1985. For some subjects, for example 'Waves on falling films' (chapter 6), this is detrimental since more recent work (see for example the survey article by $\mathbf{H}$. C. Chang, Ann. Rev. Fluid Mech. vol. 26, 1994, pp. 103-136) is not included. The core subjects of the book, 'Wave processes in gas-liquid systems' (chapter 3) and 'Wave propagation in a liquid with vapour bubbles' (chapter 4), suffer less from this because important developments took place worldwide in the period that is covered by the book.

These chapters are preceded by good introductory ones on 'Acoustics and shock 
waves in homogeneous gas- and vapour-liquid mixtures' (chapter 1) and 'Dynamics of gas and vapour bubbles' (chapter 2).

The outstanding feature of the work in Novosibirsk, as compared with that in the West, is the emphasis on thermal effects, in particular the consequences of evaporation and condensation for the propagation of pressure waves. The role of these processes in the evaluation of such waves, e.g. in vapour-liquid mixtures, is clearly exposed.

Specialists from western countries may find interesting references, so far unknown to them, in this book: from a total of 286 , about 200 are to Russian papers, which makes the book a valuable reference source. Some of the material in the book is also covered in the two volumes by another distinguished Russian scientist, R. I. Nigmatulin (Dynamics of Multiphase Media, vols 1,2, Hemisphere 1990) which, as is clear from the title, treats a wider field.

The translation from Russian to English has been done by L. Ya Yuzina, an 'English teacher at the Novosibirsk State University'. She has, no doubt, sufficient knowledge of the English language but a lack of knowledge of the subject of the book shows here and there. For example, the Russian 'opredelajetsa' may mean 'is determined by', as well as 'is defined by'. The translator uses the latter, whereas in most places the former is appropriate.

Another shortcoming is the careless way of referring to papers and books. Sometimes the authors provide the necessary details, sometimes just a name, suffering deformation more than once in the process of translation (Renkin, Gilmour, Gugonio, Vingaardt).

Nevertheless the book presents a valuable account of the work of our colleagues in Novosibirsk, in particular on thermodynamic effects on waves in two-phase flows.

L. VAN WIJNGAARDEN 